\title{
Permanence for a N-species competitive system with feedback controls
}

\author{
Qingshui Liao $^{\text {a,* }}$ \\ Guizhou Normal University, Guiyang, Guizhou, China \\ a Email:qsliao@gznu.edu.cn \\ *Qingshui Liao
}

Keywords: Permanence; Competitive system; Feedback control; Time scales.

Abstract: By applying the theory of inequality on time scales, we obtain some sufficient conditions which guarantee the permanence of the following $\mathrm{N}$-species competitive system with feedback controls

$$
\left\{\begin{array}{l}
x_{i}^{\Delta}(t)=b_{i}(t)-\sum_{j=1}^{N} a_{i j}(t) \exp \left\{x_{j}(t)\right\}-\sum_{j=1, j \neq i}^{N} c_{i j}(t) \exp \left\{x_{i}(t)+x_{j}(t)\right\}-d_{i}(t) u_{i}(t), \\
u_{i}^{\Delta}(t)=r_{i}(t)-e_{i}(t) u_{i}(t)+f_{i}(t) \exp \left\{x_{i}(t)\right\}, i=1,2, \ldots, N,
\end{array}\right.
$$

where $b_{i}(t), d_{i}(t), r_{i}(t), e_{i}(t), f_{i}(t), a_{i j}(t)$ and $c_{i j}(t)$ are all bounded non-negative almost periodic functions on $\mathbb{T}$.

\section{Introduction}

Recently, the dynamic behaviors of Lotka-Volterra predator-prey system have been widely investigated. And it is important to know the existence of periodic solutions of competitive systems. In [1], Ahmad S. has proved that under some certain conditions $x_{1}(t)$ is permanence and $x_{2}(t)$ is extinction in the following two-species system

$$
\left\{\begin{array}{l}
\dot{x}_{1}(t)=x_{1}(t)\left[r_{1}(t)-a_{1}(t) x_{1}(t)-b_{1}(t) x_{2}(t)\right] \\
\dot{x}_{2}(t)=x_{2}(t)\left[r_{2}(t)-a_{2}(t) x_{1}(t)-b_{2}(t) x_{2}(t)\right] .
\end{array}\right.
$$

In order to search for certain schemes (such as harvesting procedure) to ensure system (1.1) coexists under the conditions obtained in [1]. Xiao et al. [2] consider the following feedback controlled system

$$
\left\{\begin{array}{l}
\dot{x}_{1}(t)=x_{1}(t)\left[r_{1}(t)-a_{1}(t) x_{1}(t)-b_{1}(t) x_{2}(t)-d_{1}(t) u_{1}(t)\right], \\
\dot{x_{2}}(t)=x_{1}(t)\left[r_{2}(t)-a_{2}(t) x_{1}(t)-b_{2}(t) x_{2}(t)+d_{2}(t) u_{2}(t)\right], \\
\dot{u}_{1}(t)=-e_{1}(t) u_{1}(t)+f_{1}(t) x_{1}(t), \\
\left.\dot{u}_{2}(t)=h_{2}(t)-e_{2}(t) u_{2}(t)-f_{2}(t) x_{2}(t)\right] .
\end{array}\right.
$$

Due to the various seasonal effects of the environmental factors in real life situation (e.g. seasonal effects of weather, food supplies, mating habits, harvesting, etc.), it is rational and practical to study the ecosystem with periodic coefficients[3-7].

Up to now, few work has been done for multispecies competitive system on time scales which can unify continuous and discrete situations. In [8], the authors propose the concept of almost periodic time scales and the definition of almost periodic functions on almost periodic time scales. Based on these, our main aim in this paper is to study the permanence of the following system with feedback controls on time scales

$$
\left\{\begin{array}{l}
x_{i}^{\Delta}(t)=b_{i}(t)-\sum_{j=1}^{N} a_{i j}(t) \exp \left\{x_{j}(t)\right\}-\sum_{j=1, j \neq i}^{N} c_{i j}(t) \exp \left\{x_{i}(t)+x_{j}(t)\right\}-d_{i}(t) u_{i}(t), \\
u_{i}^{\Delta}(t)=r_{i}(t)-e_{i}(t) u_{i}(t)+f_{i}(t) \exp \left\{x_{i}(t)\right\}, i=1,2, \ldots, N .
\end{array}\right.
$$

where $t \in \mathbb{T}, \mathbb{T}$ is an almost periodic time scale, $x_{i}(t), i=1,2, \ldots, N$ is the density of species $X_{i}$; 
$u_{i}(t), i=1,2, \ldots, N$ is feedback control; $b_{t}, a_{i j}(t)$ and $c_{i j}(t)$ denote the intrinsic growth rate, death rate and inter-specific competition, respectively. $b_{i}(t), d_{i}(t), r_{i}(t), e_{i}(t), f_{i}(t), a_{i j}(t)$ and $c_{i j}(t)$ are all bounded non-negative almost periodic functions on $\mathbb{T}$.

For an almost periodic function $f: \mathbb{T} \rightarrow \mathbb{R}$, we denote $f^{M}=\sup _{t \in \mathbb{T}} f(t), f^{m}=\inf _{t \in \mathbb{T}} f(t)$, and we denote the solutions of system (1.3) by $X(t)=\left(x_{1}(t), \ldots, x_{N}(t), u_{1}(t), \ldots, u_{N}(t)\right)^{T}$.

Throughout this paper, we assume that $\left(\mathrm{H}_{1}\right) b_{i}(t), d_{i}(t), r_{i}(t), e_{i}(t), f_{i}(t), a_{i j}(t)$ and $c_{i j}(t)$ are all bounded non-negative almost periodic functions on $\mathbb{T}$ such that

$$
\begin{aligned}
& 0<b_{i}^{m} \leq b_{i}(t) \leq b_{i}^{M}, 0<d_{i}^{m} \leq d_{i}(t) \leq d_{i}^{M}, \\
& 0<r_{i}^{m} \leq r_{i}(t) \leq r_{i}^{M}, 0<e_{i}^{m} \leq e_{i}(t) \leq e_{i}^{M}, \\
& 0<f_{i}^{m} \leq f_{i}(t) \leq f_{i}^{M}, 0<a_{i j}^{m} \leq a_{i j}(t) \leq a_{i j}^{M}, \\
& 0<c_{i j}^{m} \leq c_{i j}(t) \leq c_{i j}^{M}, i, j=1,2, \ldots, N ;
\end{aligned}
$$

$\left(\mathrm{H}_{2}\right)-b_{i}^{M},-b_{i}^{m},-d_{i}^{M},-d_{i}^{m},-r_{i}^{M},-r_{i}^{m},-e_{i}^{M},-e_{i}^{m},-f_{i}^{M},-f_{i}^{m},-a_{i}^{M},-a_{i j}^{m},-c_{i}^{M},-c_{i j}^{m}, \in \boldsymbol{\mathcal { R }}^{+} \$ ;$

$\left(\mathrm{H}_{3}\right) b_{i}^{m}-\sum_{j=1, j \neq i}^{N} a_{i j}^{M} \exp \left\{x_{j}^{*}\right\}-\sum_{j=1, j \neq i}^{N} c_{i j}^{M} \exp \left\{x_{i}^{*}+x_{j}^{*}\right\}-d_{i}^{M}(t) u_{i}^{*}>0$.

\section{Preliminaries}

Let $\mathbb{T}$ be a nonempty closed subset (time scale) of $\mathbb{R}$. The forward and backward jump operators $\sigma, \rho: \mathbb{T} \rightarrow \mathbb{T}$ and the graininess $\mu: \mathbb{T} \rightarrow \mathbb{R}^{+}$are defined, respectively, by

$$
\sigma(t)=\inf \{s \in \mathbb{T}: s>t\}, \quad \rho(t)=\sup \{s \in \mathbb{T}: s<t\}, \quad \mu(t)=\sigma(t)-t .
$$

A point $t \in \mathbb{T}$ is called left-dense if $t>\inf \mathbb{T}$ and $\rho(t)=t$, left-scattered if $\rho(t)<t$, right-dense if $t<\sup \mathbb{T}$ and $\sigma(t)=t$, and right-scattered if $\sigma(t)>t$. If $\mathbb{T}$ has a left-scattered maximum $m$, then $\mathbb{T}^{k}=\mathbb{T} \backslash\{m\}$; otherwise $\mathbb{T}^{k}=\mathbb{T}$. If $\mathbb{T}$ has a right-scattered minimum $m$, then $\mathbb{T}_{k}=\mathbb{T} \backslash\{m\}$; otherwise $\mathbb{T}_{k}=\mathbb{T}$.

A function $f: \mathbb{T} \rightarrow \mathbb{R}$ is right-dense continuous provided it is continuous at right-dense point in $\mathbb{T}$ and its left-side limits exist at left-dense points in $\mathbb{T}$. If $f$ is continuous at each right-dense point and each left-dense point, then $f$ is said to be a continuous function on $\mathbb{T}$.

Definition 2.1[8] Assume that $f: \mathbb{T} \rightarrow \mathbb{R}$ is a function and let $t \in \mathbb{T}$. Then we define $f^{\Delta}(t)$ to be the number (provided it exists) with the property that given any $\varepsilon>0$, there is a neighborhood $U$ of $t$ (i.e., $U=(t-\delta, t+\delta) \cap \mathbb{T}$ for some $\delta>0)$ such that

$$
\left|[f(\sigma(t))-f(s)]-f^{\Delta}(t)[\sigma(t)-s]\right| \leq \varepsilon|\sigma(t)-s| \text { for all } s \in U
$$

we call $f^{\Delta}(t)$ the delta derivative of $f$ at $t$. The function $f$ is delta differentiable on $\mathbb{T}$ provided $f^{\Delta}(t)$ exists for all $t \in \mathbb{T}$. The set of functions $f: \mathbb{T} \rightarrow \mathbb{R}$ that are delta differentiable and whose delta derivative are rd-continuous functions is denoted by $C_{r d}^{1}=C_{r d}^{1}(\mathbb{T})=C_{r d}^{1}(\mathbb{T}, \mathbb{R})$.

Definition 2.2[8] A function $p: \mathbb{T} \rightarrow \mathbb{R}$ is called regressive provided $1+\mu(t) p(t) \neq 0$ for all $t \in \mathbb{T}^{k}$. The set of all regressive and rd-continuous functions $p: \mathbb{T} \rightarrow \mathbb{R}$ will be denoted by $\mathcal{R}=\mathcal{R}(\mathbb{T})=\mathcal{R}(\mathbb{T}, \mathbb{R})$. We define the set $\mathcal{R}^{+}=\mathcal{R}^{+}(\mathbb{T}, \mathbb{R})=\{p \in \mathcal{R}: 1+\mu(t) p(t)>0, \forall t \in \mathbb{T}\}$.

Definition 2.3[8] A time scale $\mathbb{T}$ is called an almost periodic time scale if $\Pi:=\{\tau \in \mathbb{R}: t+\tau \in \mathbb{T}, \forall t \in \mathbb{T}\} \neq\{0\}$.

Throughout this paper, we restrict our discussion on almost periodic time scales.

Definition 2.4[8] Let $\mathbb{T}$ be an almost periodic time scale. A function $f: \mathbb{T} \rightarrow \mathbb{R}^{n}$ is said to be almost periodic on $\mathbb{T}$, if for any $\varepsilon>0$, the set $E(\varepsilon, f)=\{\tau \in \Pi:|f(t+\tau)-f(t)|<\varepsilon, \forall t \in \mathbb{T}\}$ is relatively dense in $\mathbb{T}$, that is, for any $\varepsilon>0$, there exists a constant $l(\varepsilon)>0$ such that each interval of 
length $l(\varepsilon)$ contains at least one $\tau \in E(\varepsilon, f)$ such that $|f(t+\tau)-f(t)|<\varepsilon, \forall t \in \mathbb{T}$. The set $E(\varepsilon, f)$ is called the $\varepsilon$-translation set of $f(t), \tau$ is called the $\varepsilon$-translation number of $f(t)$, and $l(\varepsilon)$ is called the inclusion of $E(\varepsilon, f)$.

Lemma 2.1[8] Let $-a \in \mathcal{R}^{+}$.

(i) If $x^{\Delta}(t) \leq b-a x(t)$, then for $t>t_{0}, x(t) \leq x\left(t_{0}\right) e_{(-a)}\left(t, t_{0}\right)+\frac{b}{a}\left(1-e_{(-a)}\left(t, t_{0}\right)\right)$. In particular, if $a>0$, we have $\limsup _{t \rightarrow+\infty} x(t) \leq \frac{b}{a}$.

(ii) If $x^{\Delta}(t) \geq b-a x(t)$, then for $t>t_{0}, x(t) \geq x\left(t_{0}\right) e_{(-a)}\left(t, t_{0}\right)+\frac{b}{a}\left(1-e_{(-a)}\left(t, t_{0}\right)\right)$.In particular, if $a>0$, we have $\liminf _{t \rightarrow+\infty} x(t) \geq \frac{b}{a}$.

\section{Permanence}

In this section, we establish some permanence results for system (1.3). Firstly, we provide the definition of permanence.

Definition 3.1 System (1.3) is said to be permanent if there exist positive constants $x_{i_{*}}, x_{i}^{*}, u_{i_{*}}, u_{i}^{*}$ which are independent of the solutions of the system, such that any positive solution $X(t)$ of system (1.3) satisfies

$$
x_{i_{*}} \leq \lim \inf _{t \rightarrow+\infty} x_{i}(t) \leq \lim \sup _{t \rightarrow+\infty} x_{i}(t) \leq x_{i}^{*}, \quad u_{i_{*}} \leq \lim \inf _{t \rightarrow+\infty} u_{i}(t) \leq \lim \sup _{t \rightarrow+\infty} u_{i}(t) \leq u_{i}^{*}, \quad i=1,2, \ldots, N .
$$

Theorem 3.1 Assume that $\left(\mathrm{H}_{1}\right),\left(\mathrm{H}_{2}\right)$ hold. Then every solution $X(t)$ of system (1.3) satisfies

$$
\lim \sup _{t \rightarrow+\infty} x_{i}(t) \leq x_{i}^{*}, \lim \sup _{t \rightarrow+\infty} u_{i}(t) \leq u_{i}^{*}, \quad i=1,2, \ldots, N,
$$

where

$$
x_{i}^{*}=\frac{b_{i}^{M}-a_{i i}^{m}}{a_{i i}^{m}}, u_{i}^{*}=\frac{r_{i}^{M}+f_{i}^{M} \exp \left\{x_{i}^{*}\right\}}{e_{i}^{m}}, i=1,2, \ldots, N .
$$

Proof From the first equation of (1.3), we have

$$
\begin{aligned}
x_{i}^{\Delta}(t) & \leq b_{i}(t)-\sum_{j=1}^{N} a_{i j}(t) \exp \left\{x_{j}(t)\right\} \\
& \leq b_{i}(t)-a_{i i}(t) \exp \left\{x_{j}(t)\right\} \\
& \leq b_{i}(t)-a_{i i}(t)\left(x_{i}(t)+1\right) n \\
& \leq b_{i}^{M}-a_{i i}^{m}-a_{i i}^{m} x_{i}(t) .
\end{aligned}
$$

It follows from Lemma 2.1(i), that

$$
\lim \sup _{t \rightarrow+\infty} x_{i}(t) \leq x_{i}^{*} .
$$

Then for any $\varepsilon>0$, there exists a $t_{0} \in \mathbb{T}$ such that $x_{i}(t) \leq x_{i}^{*}+\varepsilon$ for all $t \geq t_{0}$. While, from the second equation of (1.3), we get

Letting $\varepsilon \rightarrow 0$, we get

$$
\begin{aligned}
u_{i}^{\Delta}(t) & \leq r_{i}(t)-e_{i}(t) u_{i}(t)+f_{i}(t) \exp \left\{x_{i}^{*}+\varepsilon\right\} \\
& \leq r_{i}^{M}+f_{i}^{M} \exp \left\{x_{i}^{*}+\varepsilon\right\}-e_{i}^{m} u_{i}(t) .
\end{aligned}
$$

$$
\lim \sup _{t \rightarrow+\infty} u_{i}(t) \leq u_{i}^{*} .
$$

Theorem 3.2 Assume that $\left(\mathrm{H}_{1}\right)-\left(\mathrm{H}_{3}\right)$ hold, then every solution $X(t)$ of system (1.1) satisfies

$$
\lim \inf _{t \rightarrow+\infty} x_{i}(t) \geq x_{i_{*}}, \lim \inf _{t \rightarrow+\infty} u_{i}(t) \geq u_{i_{*}},
$$

where 


$$
\begin{aligned}
& x_{i_{*}}=\ln \frac{b_{i}^{m}-\sum_{j=1, j \neq i}^{N} a_{i j}^{M} \exp \left\{x_{j}^{*}\right\}-\sum_{j=1, j \neq i}^{N} c_{i j}^{M} \exp \left\{x_{i}^{*}+x_{j}^{*}\right\}-d_{i}^{M} u_{i}^{*}}{a_{i i}^{M}}, \\
& u_{i_{*}}=\frac{r_{i}^{m}+e_{i}^{m} x_{i_{*}}}{e_{i}^{M}} .
\end{aligned}
$$

Proof According to Theorem 3.1, for any $\varepsilon>0$, there exists a $t_{0} \in \mathbb{T}$, for any $t>t_{0}$ and $t \in \mathbb{T}$ such that, $u_{i}(t) \leq x_{i}^{*}+\varepsilon, x_{i}(t) \leq u_{i}^{*}+\varepsilon$. Then for $t \geq t_{0}$, from the first equation of (1.3), we have

$$
\begin{aligned}
x_{i}^{\Delta}(t)= & b_{i}(t)-\sum_{j=1, j \neq i}^{N} a_{i j} \exp \left\{x_{j}(t)\right\}-\sum_{j=1, j \neq i}^{N} c_{i j} \exp \left\{x_{i}(t)+x_{j}(t)\right\} \\
& -d_{i}(t) u_{i}(t)-a_{i i}(t) \exp \left\{x_{i}(t)\right\} \\
\geq & b_{i}^{m}-\sum_{j=1, j \neq i}^{N} a_{i j}^{M} \exp \left\{x_{j}^{*}+\varepsilon\right\}-\sum_{j=1, j \neq i}^{N} c_{i j}^{M} \exp \left\{x_{i}^{*}+x_{j}^{*}+2 \varepsilon\right\} \\
& -d_{i}^{M}(t) u_{i}^{*}-a_{i i}^{M} \exp \left\{x_{i}(t)\right\} .
\end{aligned}
$$

Now, we claim for any $\varepsilon>0$, there exists a $t_{0} \in \mathbb{T}$, for any $t>t_{0}$ and $t \in \mathbb{T}$ such that,

$$
\begin{aligned}
N(t) & =b_{i}^{m}-\sum_{j=1, j \neq i}^{N} a_{i j}^{M} \exp \left\{x_{j}^{*}+\varepsilon\right\}-\sum_{j=1, j \neq i}^{N} c_{i j}^{M} \exp \left\{x_{i}^{*}+x_{j}^{*}+2 \varepsilon\right\}-d_{i}^{M}(t) u_{i}^{*}-a_{i i}^{M} \exp \left\{x_{i}(t)\right\} \\
& \leq 0,
\end{aligned}
$$

where

$$
x_{i}(t) \geq \ln \frac{b_{i}^{m}-\sum_{j=1, j \neq i}^{N} a_{i j}^{M} \exp \left\{x_{j}^{*}+\varepsilon\right\}-\sum_{j=1, j \neq i}^{N} c_{i j}^{M} \exp \left\{x_{i}^{*}+x_{j}^{*}+2 \varepsilon\right\}-d_{i}^{M}(t) u_{i}^{*}}{a_{i i}^{M}} .
$$

Otherwise, there exists a $t_{1}>t_{0}$ and $t_{1} \in \mathbb{T}$, when $t \geq t_{1}$, such that,

$$
\begin{aligned}
N_{0}(t) & =b_{i}^{m}-\sum_{j=1, j \neq i}^{N} a_{i j}^{M} \exp \left\{x_{j}^{*}+\varepsilon\right\}-\sum_{j=1, j \neq i}^{N} c_{i j}^{M} \exp \left\{x_{i}^{*}+x_{j}^{*}+2 \varepsilon\right\}-d_{i}^{M}(t) u_{i}^{*}-a_{i i}^{M} \exp \left\{x_{i}(t)\right\} \\
& >0,
\end{aligned}
$$

where

$$
x_{i}\left(t_{1}\right)<\ln \frac{\left.b_{i}^{m}-\sum_{j=1, j \neq i}^{N} a_{i j}^{M} \exp \left\{x_{j}^{*}+\varepsilon\right\}-\sum_{j=1, j \neq i}^{N} c_{i j}^{M} \exp \left\{x_{i}^{*}+x_{j}^{*}+2 \varepsilon\right\}-d_{i}^{M}(t) u_{i}^{*}\right\}}{a_{i i}^{M}},
$$

and for $t \in\left[t_{0}, t_{1}\right)_{\mathbb{T}}, \quad N(t) \leq 0$.

From (3.3) and (3.5), we get

But from $\left(\mathrm{H}_{3}\right)$ and (3.1) we have

$$
x_{i}^{\Delta}\left(t_{1}\right) \leq 0
$$

which is a contradiction with (3.6). Thus, we have proved the claim.

Then, from (3.2), we arrive that

$$
x_{i}(t) \geq \ln \frac{\left.b_{i}^{m}-\sum_{j=1, j \neq i}^{N} a_{i j}^{M} \exp \left\{x_{j}^{*}+\varepsilon\right\}-\sum_{j=1, j \neq i}^{N} c_{i j}^{M} \exp \left\{x_{i}^{*}+x_{j}^{*}+2 \varepsilon\right\}-d_{i}^{M}(t) u_{i}^{*}\right\}}{a_{i i}^{M}} .
$$

Letting $\varepsilon \rightarrow 0$, we get $\lim _{t \rightarrow+\infty} x_{i}(t) \geq x_{i_{*}}$.

For any $\varepsilon>0$, there exists a $t_{2} \in \mathbb{T}$, for any $t>t_{2}$ and $t \in \mathbb{T}$ such that, $x_{i}(t) \leq x_{i_{*}}-\varepsilon$, for $t \geq t_{2}$. Owing to the second equation of system (1.3) we get,

$$
\begin{aligned}
u_{i}^{\Delta}(t) & =r_{i}(t)-e_{i}(t) u_{i}(t)+f_{i}(t) x_{i}(t) \\
& \geq r_{i}^{m}+f_{i}^{m}\left(x_{i_{*}}-\varepsilon\right)-e_{i}^{M} u_{i}(t)
\end{aligned} .
$$


Letting $\varepsilon \rightarrow 0$, we get

$$
u_{i}^{\Delta}(t) \geq r_{i}^{m}+f_{i}^{m} x_{i_{*}}-e_{i}^{M} u_{i}(t),
$$

according to Lemma 2.1(i), it follows that,

$$
\lim \inf _{t \rightarrow+\infty} u_{i}(t) \geq u_{i_{*}}
$$

Obviously, we can obtain the following result.

Theorem 3.3 Assume that $\left(\mathrm{H}_{1}\right)-\left(\mathrm{H}_{3}\right)$ hold. Then system (1.3) is permanent.

We denote by $\Omega$ for all solutions $X(t)$ of system (1.3) satisfying $x_{i_{*}} \leq x_{i}(t) \leq x_{i_{*}}, \quad u_{i_{*}} \leq u_{i}(t) \leq u_{i_{*}}$, for $t \in \mathbb{T}, \quad i=1,2, \ldots, N$.

Theorem 3.4 Assume $\left(\mathrm{H}_{1}\right)-\left(\mathrm{H}_{3}\right)$ hold, then $\Omega \neq \varnothing$.

Proof Since $b_{i}(t), d_{i}(t), r_{i}(t), e_{i}(t), f_{i}(t), a_{i j}(t)$ and $c_{i j}(t)$ all are almost periodic functions on $\mathbb{T}$, then there exists a sequence $\tau=\left\{\tau_{p}\right\} \subseteq \mathbb{T}$ with $\tau_{p} \rightarrow+\infty$ for $p \rightarrow+\infty$ such that

$$
\begin{aligned}
& b_{i}\left(t+\tau_{p}\right) \rightarrow b_{i}(t), d_{i}\left(t+\tau_{p}\right) \rightarrow d_{i}(t), r_{i}\left(t+\tau_{p}\right) \rightarrow r_{i}(t), \\
& e_{i}\left(t+\tau_{p}\right) \rightarrow e_{i}(t), f_{i}\left(t+\tau_{p}\right) \rightarrow f_{i}(t), a_{i j}\left(t+\tau_{p}\right) \rightarrow a_{i j}(t), \\
& a_{i j}\left(t+\tau_{p}\right) \rightarrow a_{i j}(t), \text { for } p \rightarrow+\infty .
\end{aligned}
$$

According to the Theorem 3.1 and Theorem 3.2, for any $\varepsilon>0$, there exists a $t_{+} \in \mathbb{T}$ such that

$$
x_{i_{*}}-\varepsilon \leq x_{i}(t) \leq x_{i}^{*}+\varepsilon, \quad u_{i_{*}}-\varepsilon \leq u_{i}(t) \leq u_{i}^{*}+\varepsilon, \text { for all } t \geq t_{+} .
$$

For $t>t_{+}-\tau_{p}, p=1,2, \ldots, N$, we denote $x_{i p}(t)=x_{i}\left(t+\tau_{p}\right)$ and $u_{i p}(t)=u_{i}\left(t+\tau_{p}\right)$. For any positive integer $q$, it is easy to see that there exist sequences $\left\{x_{i p}(t): p \geq q\right\}$ and $\left\{u_{i p}(t): p \geq q\right\}$ such that the sequences $\left\{x_{i p}(t)\right\}$ and $\left\{i_{i p}(t)\right\}$ have subsequences, converging on any finite interval of $\mathbb{T}$ for $p \rightarrow+\infty$, respectively. For the convenience of expression, we denoted by $\left\{x_{i p}(t)\right\}$ and $\left\{u_{i p}(t)\right\}$ again. Thus we have sequences $\left\{m_{i}(t)\right\}$ and $\left\{n_{i}(t)\right\}$ such that

$$
x_{i p}(t) \rightarrow m_{i}(t), u_{i p}(t) \rightarrow n_{i}(t) \text {, for } p \rightarrow+\infty \text {. }
$$

Combining with

$$
\left\{\begin{aligned}
x_{i p}^{\Delta}(t)= & b_{i}\left(t+\tau_{p}\right)-\sum_{j=1}^{N} a_{i j}\left(t+\tau_{p}\right) \exp \left\{x_{i p}(t)\right\}-d_{i}\left(t+\tau_{p}\right) u_{i p}(t) \\
& -\sum_{j=1, j \neq i}^{N} c_{i j}\left(t+\tau_{p}\right) \exp \left\{x_{j p}(t)+x_{i p}(t)\right\}, \\
u_{i p}^{\Delta}(t)= & r_{i}\left(t+\tau_{p}\right)-e_{i}\left(t+\tau_{p}\right) u_{i p}(t)+f_{i}\left(t+\tau_{p}\right) \exp \left\{x_{i p}(t)\right\},
\end{aligned}\right.
$$

from (3.7) and (3.8), for $p \rightarrow+\infty$, (3.9) arrives to

$$
\left\{\begin{aligned}
m_{i}^{\Delta}(t)= & b_{i}(t)-\sum_{j=1}^{N} a_{i j}(t) \exp \left\{m_{i}(t)\right\}-d_{i}(t) n_{i}(t) \\
& -\sum_{j=1, j \neq i}^{N} c_{i j}(t) \exp \left\{m_{j}(t)+m_{i}(t)\right\}, \\
n_{i}^{\Delta}(t)= & r_{i}(t)-e_{i}(t) n_{i}(t)+f_{i}(t) \exp \left\{m_{i}(t)\right\} .
\end{aligned}\right.
$$

We can easily see that the solution of (3.10)

$$
Y(t)=\left(m_{1}(t), m_{2}(t), \ldots, m_{N}(t), n_{1}(t), n_{2}(t), \ldots, n_{N}(t)\right)^{T},
$$

is a solution of system (1.3), moreover for any $\varepsilon>0$ and for all $t \in \mathbb{T}$ meet the following consideration

$$
x_{i_{*}}-\varepsilon \leq m_{i}(t) \leq x_{i}^{*}+\varepsilon, u_{i_{*}}-\varepsilon \leq n_{i}(t) \leq u_{i}^{*}+\varepsilon .
$$

Since $\varepsilon$ is an arbitrary small positive number, it follows that $x_{i_{*}} \leq m_{i}(t) \leq x_{i}^{*}, u_{i_{*}} \leq n_{i}(t) \leq u_{i}^{*}$, for all $t \in \mathbb{T}$. 


\section{References}

[1] Ahmad, S. (1993) On nonautonomous Volterra-Lotka competition equations. Proc. Amer. Math. Soc. $177(1), 199-204$.

[2] Xia, Y.H., Chen, F.D. (2004) Almost periodic solutions of n-species competitive system with feedback controls. J. Math. Anal. Appl., 294(2), 503-522.

[3] Liao, Q.S., Li, B., Li, Y.K. (2015) Permanence and almost periodic solutions for an n-species Lotkau-Volterra food chain system on time scales. Asian-European J. Math., 8(02), 1152-1160.

[4] Geng, J.B., Xia, Y.H. (2011) Almost periodic solutions of a nonlinear ecological model, Commun. Nonlinear Sci., 16, 2575-2597.

[5] Lou, Y., Ni, W.M., Yotsutani, S. (2017) On a limiting system in the Lotka-Volterra competition with cross- diffusion. Discret. Contin. Dyn. S., 37(8), 435-458.

[6] Liu, M., Fan, M. (2017) Permanence of stochastic Lotka-Volterra systems. J. Nonlinear. Sci., 27(2), 425-452.

[7] Xiao, Y., Tang, S., Chen, J. (1998) Permanence and periodic solution in competitive system with feedback controls. Math. Comput. Model., 27(6), 33-37.

[8] Li, Y.K., Wang, C. (2011) Uniformly Almost Periodic Functions and Almost Periodic Solutions to Dynamic Equations on Time Scales. Abstract and Applied Analysis, Article ID 341520, 23 pages. 